\title{
La demanda insatisfecha en los proyectos de inversión pública
}

\begin{abstract}
RESUMEN
La demanda insatisfecha es uno de los aspectos más importantes que se desarrollan en la elaboración de los proyectos de inversión, su presentación y determinación adecuada ayuda a sustentar la intervención y los recursos necesarios para implementarlo. El presente artículo ilustra conceptualmente la forma correcta de su presentación.

Palabras clave: demanda insatisfecha, inversión, proyectos

The UnMet Demand IN PUBLIC INVESTMENT PROJECTS

\section{ABSTRACT}

The unmet demand is one of the most important side in the investment projects, their presentation and proper determination helps support the involvement and investment. This article illustrates conceptually the best form of submission.
\end{abstract}

Keywords: unmet demand, investment, projects

\section{INTRODUCCIÓN}

Una vez identific123 1 ado el problema de la situación a enfrentar a través de un proyecto de inversión es necesario determinar su magnitud, es decir, cuantificar el nivel de intervención en cuanto a cobertura, para ello se debe estimar a quienes va a ser dirigido el proyecto y la cantidad de bienes o servicios que se va a entregar (equipos, materiales de capacitación, número de talleres, cursos de capacitación, etc.). Dicha cuantificación se debe realizar en los proyectos de inversión pública para determinar los recursos necesarios que se van a requerir. Es decir, una vez identificado el problema de un proyecto en la formulación se cuantifica, luego se determina los recursos necesarios, los que forman la inversión del proyecto.

La cuantificación se debe realizar en el ámbito definido en la identificación y debe tener relación con el problema, por lo que su magnitud debe expresar dicha realidad, ya sea en una sola unidad de medida o en varias. Es importante resaltar que la cuantificación de la necesidad define y brinda la información para determinar la inversión del proyecto.

Es por ello que toma relevancia el determinar en forma correcta la demanda insatisfecha de un proyecto, por lo que el presente artículo tiene por objetivo precisar los conceptos y enfoques en esta temática.

\section{PROYECTO DE INVERSIÓN}

El proyecto de inversión desde una perspectiva general, se entiende como una intervención en un medio para dar solución a una problemática existente y lograr un cambio deseado, por lo que va a existir un grupo de personas involucradas y necesidades referida a la problemática.

El enfoque de desarrollo de un proyecto de inversión se inicia con la identificación del problema, luego se cuantifica, en función a ello se determina los requerimientos, cuya valorización se convierte en la inversión del proyecto, esquema que se muestra en la Figura $\mathrm{N} .^{\circ} 1$.

1 Magíster en Gestión Económica y Empresarial, Ingeniero Industrial - UNMSM, Profesor en la Facultad de Ingeniería Industrial, Departamento Académico de Producción y Gestión Industrial de la UNMSM.

Email: cieandia@hotmail.com 
Figura 1

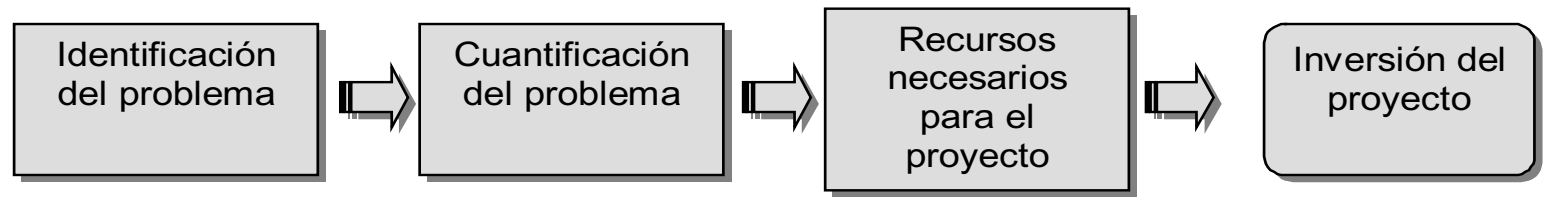

Fuente: Elaboración propia

\section{Demandantes y demanda}

En la cuantificación del problema es necesario diferenciar los conceptos de demanda y demandantes. Los demandantes son aquellas personas y/o instituciones que tienen la necesidad identificada por el proyecto y la cuantificación de dicha necesidad es la demanda.

Entonces, se debe diferenciar la demanda de los demandantes, la primera refiere la necesidad expresada en información, bienes y/o otros servicios, el segundo son los usuarios que requieren el servicio.

Por ejemplo: cuando se trata temas de capacitación, la demanda se puede expresar en horas de capacitación y los demandantes serían las personas que lo requieren.

La demanda tiene por objetivo cuantificar la necesidad identificada por el proyecto, esta cuantificación se debe expresar en una unidad que lleve los siguientes criterios [2]:

- La unidad de medida debe representar la necesidad.

- La unidad de medida debe ser acumulable.

Figura 2

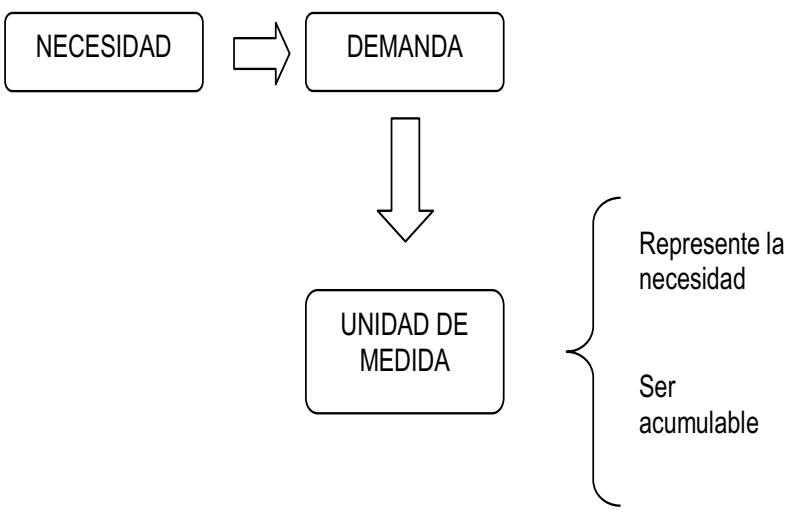

Fuente: Elaboración propia

\section{Demanda}

La estimación de la demanda se realiza considerando la cantidad de la población objetivo y sus necesidades en un horizonte de tiempo.

Para determinar correctamente la demanda es necesario realizar las siguientes interrogantes:

1.- ¿Quién demanda?

2.- ¿Qué demanda?

¿Cómo se puede expresar?

¿Cuál es el componente principal? (Se utiliza principalmente en servicios).

3.- ¿Cuánto demandan?

Ejemplos:

Para un proyecto de un hotel turístico:

1) ¿Quién demanda?

Turistas

2) ¿Qué demanda?

Servicio de hospedaje

Se expresa en días de estadía

Componente principal: número de habitaciones

3) ¿Cuánto demandan?

200 habitaciones por mes.

Para un proyecto de un colegio:

1) ¿Quién demanda?

250 niños

2) ¿Qué demanda?

Servicio educativo

Se expresa en cantidad de aulas

3) ¿Cuánto demandan?

8 aulas.

Para un proyecto de una carretera:

1) ¿Quién demanda?

Pasajeros, transportistas, etc.

2) ¿Qué demanda?

Vía 
Se expresa en $\mathrm{km}$ de distancia

3) ¿Cuánto demandan?

$200 \mathrm{~km}$ del punto $A$ al $B$.

Para un proyecto de desarrollo de capacidades en jóvenes:

1) ¿Quién demanda?

200 jóvenes

2) ¿Qué demanda?

Acceso a información de formación productiva

Se expresa en cantidad de horas de capacitación

3) ¿Cuánto demandan?

40 horas

El análisis se debe realizar para cada componente del proyecto, es decir, si un proyecto tiene tres componentes (obra, equipamiento, capacitación, etc.), para cada uno de ellos se tiene que determinar su demanda. La elaboración de los proyectos por sus componentes tiene un carácter multidisciplinario, por lo tanto, en su elaboración deben participar distintos especialistas, quienes con su enfoque aportarán elementos conceptuales para sustentar los aspectos técnicos del proyecto.

\section{Oferta}

Es la capacidad de servicio existente y está relacionado al problema identificado, expresada en la misma unidad de medida que la demanda.

\section{DEMANDA INSATISFECHA}

Término usado en todo proyecto que engloba los siguientes conceptos [3]:

\section{Demanda no atendida}

Es aquella en donde parte de una población o un conjunto de instituciones no reciben el servicio y/o producto que requieren, por lo tanto, la demanda es mayor que la oferta. El proyecto cubrirá una porción o la totalidad de la brecha identificada.

\section{Demanda atendida}

Es aquella donde se brinda el servicio y/o producto a casi la totalidad del mercado, pero se satisface en forma parcial la necesidad identificada, por lo que también representa una demanda insatisfecha. Aquí el proyecto debe brindar un nuevo servicio que incorpore esos requerimientos, por lo tanto, la oferta actual del nuevo servicio sería nula.

Entonces, la "demanda insatisfecha» es aquella que incluye una de las demandas antes mencionadas o su combinación; en este caso, el proyecto cubrirá la demanda no atendida y/o la demanda atendida pero no satisfecha.

En términos operativos la comparación de lo que se requiere (demanda) y lo que se está satisfaciendo (oferta) es la demanda insatisfecha, por lo que ambas deben estar expresadas en las mismas unidades. El proyecto con sus recursos debe cubrir dicha demanda insatisfecha. Por ejemplo, para una biblioteca comunal, la demanda del componente material bibliográfico estaría representada por la cantidad de textos, la oferta sería cero si no existen dichos materiales, por lo que la demanda insatisfecha sería igual a la demanda.

Figura 3

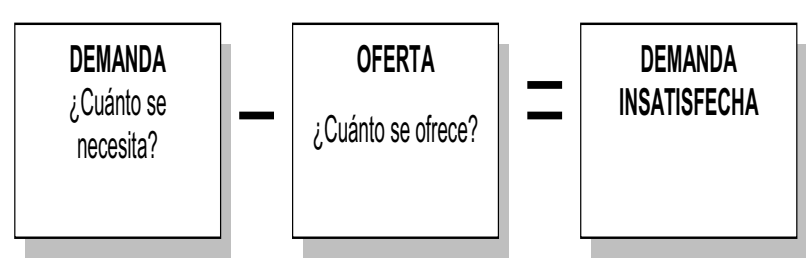

Fuente: Elaboración propia

\section{PROCEDIMIENTOS PARA LA DETERMINACIÓN DE LA DEMANDA INSATISFECHA}

\section{Demanda}

Para determinar la demanda futura de un servicio es importante diferenciar dos conceptos:

Demanda normal: es la demanda futura que el servicio tendrá sin la intervención del proyecto (demanda sin proyecto), para ello se considera las variables de la información histórica y otras complementarias.

Demanda generada: es la demanda generada por la intervención del proyecto (demanda con proyecto), para ello se considera variables adicionales que reflejen el incentivo por el uso de un servicio diferente.

Por tanto, la demanda futura de un proyecto incluye la demanda normal y la demanda generada.

$$
\begin{gathered}
\text { Demanda Futura }=\text { Demanda normal }+ \\
\text { Demanda generada }
\end{gathered}
$$

\section{Oferta}

Oferta optimizada: es la capacidad de servicio resultado de mejoras en términos de optimización de la situación actual (oferta sin proyecto). En algunos casos se presenta la oferta con proyecto, la cual no 
tiene sentido, porque lo que se desea demostrar es existencia de una demanda insatisfecha y el proyecto tiene que cubrir la misma, caso contrario se presentaría fraccionamiento.

Demanda Insatisfecha = Demanda Futura Oferta optimizada

\section{APLICACIÓN EN LOS PROYECTOS DE INVE- SIÓN PÚBLICA}

En nuestro país se ha implementado desde el año 2000 el Sistema Nacional de Inversión Pública el que administra una base de datos donde se encuentran los resúmenes de todos los proyectos de inversión elaborados por los tres niveles de gobierno: nacional, regional y local. Para la aplicación de la propuesta conceptual se han analizado los proyectos de inversión cuyo monto de inversión es menor a S/. 1200 000, denominados "Proyectos de Inversión Pública menor". Se ha encontrado los siguientes casos sobre la demanda insatisfecha, considerando los proyectos viables (aprobados y expeditos para su ejecución) durante el 2010 y 2011 [4]:

\section{Sector Transportes}

Los proyectos de inversión pública desarrollados y presentados en el Sector Transportes referido a construcción y/o reparación de vías tienen la característica común en la metodología de determinación de la demanda. El procedimiento actual según las guías metodológicas del sector es cuantificar la cantidad y tipo de flujo vehicular en ciertos puntos de la vía para luego con dicha información determinar el índice de tráfico diario, cuyo resultado es la demanda; en la oferta se describe las características de la vía existente. En muchos casos la demanda y la oferta se expresan en unidades de flujo vehicular y población beneficiaria tal como se muestra en los Cuadros 1 y 2.

Según el análisis realizado a la base de datos del Banco de proyectos, se ha encontrado que el $60 \%$ de los proyectos tienen errores en la presentación de la demanda insatisfecha.

Para el caso de proyectos de inversión pública referido a veredas se presenta la demanda insatisfecha en usuarios y tiene valores negativos (Cuadro 3).

Tanto las personas y la cantidad de vehículos no son unidades adecuadas de demanda porque no expresan la necesidad de construcción y/o reparación de vías terrestres. Además, los valores negativos no tienen un significado lógico de demanda insatisfecha.
Cuadro 1

\begin{tabular}{cc}
\hline Descripción & Flujo vehicular \\
\hline UM & Und. \\
\hline Año 1 & -9 \\
\hline Año 2 & -8 \\
\hline Año 3 & -8 \\
\hline Año 4 & -9 \\
\hline Año 5 & -9 \\
\hline Año 6... & -9 \\
\hline
\end{tabular}

Fuente: Banco de Proyectos SNIP. Elaboración propia

\section{Cuadro 2}

\begin{tabular}{ccc}
\hline $\begin{array}{c}\text { Descripción } \\
\text { UM }\end{array}$ & $\begin{array}{c}\text { Población } \\
\text { beneficiaria } \\
\text { Habitantes }\end{array}$ & $\begin{array}{c}\text { Tráfico de carros } \\
\text { Und. }\end{array}$ \\
\hline Año 1 & -448 & -30 \\
\hline Año 2 & -454 & -45 \\
\hline Año 3 & -458 & -50 \\
\hline Año 4 & -462 & -55 \\
\hline Año 5 & -470 & -60 \\
\hline Año 6... & -474 & -56 \\
\hline
\end{tabular}

Fuente: Banco de Proyectos SNIP. Elaboración propia

Cuadro 3

\begin{tabular}{cc}
\hline Descripción & $\begin{array}{c}\text { Infraestructura vial } \\
\text { y peatonal }\end{array}$ \\
\hline UM & Usuarios \\
\hline Año 1 & -540 \\
\hline Año 2 & -540 \\
\hline Año 3 & -540 \\
\hline Año 4 & -540 \\
\hline Año 5 & -540 \\
\hline Año 6... & -540 \\
\hline
\end{tabular}

Fuente: Banco de Proyectos SNIP. Elaboración propia

\section{Sector Saneamiento}

En los proyectos de agua y alcantarillado, en algunos casos la demanda se presenta en unidades de población y viviendas afectadas. En la mayoría de los casos la demanda insatisfecha se presenta en unidades de volumen, como se muestra en el Cuadro 5. El primer caso no es correcto por los 
conceptos teóricos referidos anteriormente, para el segundo caso es necesario complementar la demanda insatisfecha con unidades de medida de infraestructura ya sea de captación, trasmisión y tratamiento para que ayude a determinar la inversión.

\section{Cuadro 4}

\begin{tabular}{ccc}
\hline $\begin{array}{c}\text { Descripción } \\
\text { UM }\end{array}$ & $\begin{array}{c}\text { Población } \\
\text { beneficiaria } \\
\text { habitantes }\end{array}$ & $\begin{array}{c}\text { Viviendas } \\
\text { Afectadas } \\
\text { Und. }\end{array}$ \\
\hline Año 1 & -330 & -55 \\
\hline Año 2 & -342 & -57 \\
\hline Año 3 & -354 & -59 \\
\hline Año 4 & -365 & -61 \\
\hline Año 5 & -378 & -63 \\
\hline Año 6... & -414 & -65 \\
\hline
\end{tabular}

Fuente: Banco de Proyectos SNIP. Elaboración propia

\section{Cuadro 5}

\begin{tabular}{cc}
\hline $\begin{array}{c}\text { Descripción } \\
\text { UM }\end{array}$ & $\begin{array}{c}\text { Agua potable } \\
\text { litros / día }\end{array}$ \\
\hline Año 1 & -35400 \\
\hline Año 2 & -35600 \\
\hline Año 3 & -37540 \\
\hline Año 4 & -38540 \\
\hline Año 5 & -39740 \\
\hline Año 6... & -40620 \\
\hline Fuente: Banco de Proyectos SNIP. Elaboración propia
\end{tabular}

\section{Sector Educación}

Es común que en el sector educación los proyectos de inversión pública muestren en la demanda insatisfecha como unidades de medida a los alumnos atendidos, ello no es correcto porque son los demandantes, los demás componentes del Cuadro 6 si son correctos.

Según análisis de una muestra de proyectos de inversión pública a nivel nacional relacionados a educación inicial, cuya inversión son menores a S/. 1200 000, se ha encontrado que el 76\% presentan información de la demanda insatisfecha incorrecta.

\section{Cuadro 6}

\begin{tabular}{ccccc}
\hline $\begin{array}{c}\text { Descrip- } \\
\text { ción }\end{array}$ & $\begin{array}{c}\text { Alumnos } \\
\text { atendidos }\end{array}$ & $\begin{array}{c}\text { Infraestruc- } \\
\text { tura Escolar }\end{array}$ & $\begin{array}{c}\text { Mobiliario } \\
\text { escolar }\end{array}$ & $\begin{array}{c}\text { Infraestructura } \\
\text { Complemen- } \\
\text { taria }\end{array}$ \\
UM & Alumnos & Aulas & Módulo & Ambientes \\
\hline Año 1 & -130 & -5 & -20 & -5 \\
\hline Año 2 & -142 & -5 & -20 & -5 \\
\hline Año 3 & -154 & -5 & -20 & -5 \\
\hline Año 4 & -165 & -6 & -20 & -5 \\
\hline Año 5 & -178 & -6 & -20 & -5 \\
\hline Año 6... & -214 & -7 & -20 & -5 \\
\hline
\end{tabular}

Fuente: Banco de Proyectos SNIP. Elaboración propia

\section{Otros Sectores}

Los siguientes casos muestran un error muy común en la determinación de la demanda insatisfecha de los proyectos de inversión, es decir, considerar a la población como unidad de la demanda, el Cuadro 7 se refiere a la presentación de la demanda de un proyecto referido a un local multiusos, el Cuadro 8 a un proyecto referido a un campo deportivo y el Cuadro 9 a un proyecto referido al mejoramiento de una plazuela.

\section{Cuadro 7}

\begin{tabular}{cc}
\hline $\begin{array}{c}\text { Descripción } \\
\text { UM }\end{array}$ & $\begin{array}{c}\text { Población } \\
\text { Personas }\end{array}$ \\
\hline Año 1 & -1500 \\
\hline Año 2 & -1520 \\
\hline Año 3 & -1540 \\
\hline Año 4 & -1560 \\
\hline Año 5 & -1570 \\
\hline Año 6... & -1590 \\
\hline
\end{tabular}

Fuente: Banco de Proyectos SNIP. Elaboración propia

Cuadro 8

\begin{tabular}{cc}
\hline $\begin{array}{c}\text { Descripción } \\
\text { UM }\end{array}$ & $\begin{array}{c}\text { Campo deportivo } \\
\text { Beneficiarios }\end{array}$ \\
\hline Año 1 & -28 \\
\hline Año 2 & -39 \\
\hline Año 3 & -40 \\
\hline Año 4 & -60 \\
\hline Año 5 & -157 \\
\hline Año 6... & -190 \\
\hline
\end{tabular}

Fuente: Banco de Proyectos SNIP. Elaboración propia 
Cuadro 9

\begin{tabular}{cc}
\hline $\begin{array}{c}\text { Descripción } \\
\text { UM }\end{array}$ & $\begin{array}{c}\text { Población demandante } \\
\text { Habitantes }\end{array}$ \\
\hline Año 1 & -688 \\
\hline Año 2 & -699 \\
\hline Año 3 & -740 \\
\hline Año 4 & -760 \\
\hline Año 5 & -765 \\
\hline Año 6... & -790 \\
\hline
\end{tabular}

Fuente: Banco de Proyectos SNIP. Elaboración propia

En todos los casos los proyectos analizados han utilizado en formato denominado "PIP menor" o perfil simplificado, lo cual es inadecuado porque genera valores negativos para la demanda insatisfecha.

\section{CONCLUSIONES}

1. En los proyectos de inversión pública la demanda insatisfecha expresa la necesidad en unidades adecuadas de medida por lo que no debe referirse a la población demandante.

2. La demanda insatisfecha justifica la intervención de un proyecto por eso siempre debe expresarse en valores positivos de lo contrario no existiría necesidades.
3. El $60 \%$ de los proyectos de inversión viables en el año 2010 en el sector de transportes relacionados a vías urbanas e inversión menor a S/. 1200000 tienen errores en la presentación de la demanda insatisfecha.

4. El $76 \%$ de los proyectos de inversión viables en el año 2010 en el sector de Educación relacionados a educación inicial e inversión menor a S/. 1200000 tienen errores en la presentación de la demanda insatisfecha.

5. El formato para la elaboración de proyectos denominados "Proyecto de Inversión Pública menores" es inadecuado porque induce a valores negativos en la demanda insatisfecha, por lo que es recomendable modificarlo.

\section{REFERENCIAS BIBLIOGRÁFICAS}

[1] Andía Valencia, W. (2010). Gerencia de Proyectos Sociales y de Inversión Social. El Saber Editores. Perú.

[2] Andía Valencia, W. (2009). Proyectos de Inver-

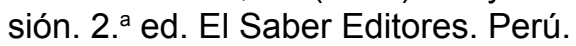

[3] Andía Valencia, W. (2011). Manual de Proyectos para el Sector Público. El Saber Editores. Perú.

[4] Ministerio de Economía y Finanzas, (2011). Guías Metodológicas para la elaboración de Proyectos de Inversión. Perú 